\title{
POSSIBILITIES OF ANALYZING THE STRUCTURE OF LANCE OUTFLOWS FROM THE LANCES
}

\author{
Ladislav KOVÁŘ, Pavel NOVÁK, Tomáš HAPLA, Jiří FRIES \\ VSB - Technical University of Ostrava, Faculty of Mechanical Engineering, 17. listopadu 15, 70833 Ostrava- \\ Poruba, Czech Republic, EU, \\ ladislav.kovar@vsb.cz,pavel.novak.st1@vsb.cz, tomas.hapla@vsb.cz, iiri.fries@vsb.cz
}

https://doi.org/10.37904/metal.2020.3441

\begin{abstract}
The design of the individual components of the systems with lances for high-temperature reactors significantly influences their proper functioning and the course of the technological process on which the quality of the resulting product depends. The lance systems intended for the high-temperature reactors are designed for gas blowing of various, especially gaseous media. From their proper functioning, they are probably the most critical elements through which the medium is directly blown to the reactor workspace, i.e. the lances. The lances provide the required parameters of the blown medium directly in the reactor working area and thus the correct course of the ongoing technological process. For the correct operation of the lances, it is important not only to comply with the required parameters of the supplied medium but above all pay attention to the design of the lance itself (the blowing element), which ensures the required parameters and functions of the lance. As for the dimensions of the lances, we are able to design these based on calculation relationships that are based on the fluid flows and their relations. However, feedback is very important for designers, i.e. verifying whether the designed lance works as the designer intended it to work. From this point of view, the designers are helped by experimental research, in which it is possible to visualize these lance outflows and, if necessary, measure the assessing quantities of currents outflowing from the lances. This paper focuses on the possibilities to analyse the structure of gas outflow jet employing the gas flow visualization.
\end{abstract}

Keywords: Gas blowing, lance outflows, lance, gas flow visualization

\section{INTRODUCTION}

To analyse the structure of the outflow jet from the lance head, in addition to knowledge of data on changes in flow parameters obtained numerically, the experimental research is vital, providing both the possibility of feedback and verification of the designed lance structure and its functionality and necessary information for the design and processing of the lance. This experimental research consists of measurements provided for the evaluative quantities which characterize these currents and from gas flow visualization.

Depending on the nature of the individual determining quantities layout, it is possible, e.g. when we consider a metallurgical heat aggregate, determine the dynamic effect of the jet on the workspace (bath), change in the concentration of the blown gas and the most suitable position of the lance to increase its service life while reducing the wear of the lining in the corresponding heat aggregate. On the outflow jets flowing from the lances, we can analyse parameters significantly influencing the lance or nozzle design intended for a given purpose, for example:

- Interaction of outflow jets in multi-hole lances,

- Length of the supersonic current core,

- The angle of outflow jets extension depending on a nozzle geometry,

- Effect of the nozzle shape on the outflow jet parameters, 
- Profiling the nozzles.

The perfect qualitative picture of free outflow jets is offered by methods of gas flow visualization. By analysing shadow and schlieren images, characteristic cross-sections can be determined, in which we then can determine the distribution of the assessment quantities using quantitative contact measurement, or using optical methods such as traditional interferometry or holographic interferometry [1]. Experimental measurements can be carried out on models, but also on the real system. Measurement on models allows you to analyze various alternative solutions with significantly lower costs. By comparing measurements on models with real-system measurements, it has been shown that the results of model research can be fully applied to the real system.

\section{METHODS APPLICABLE FOR THE GAS FLOW VISUALIZATION}

For visualization of gas outflow jets, the optical visualization methods are used with many benefits, which are used in many other sectors of research activities. These methods are particularly advantageous because the phenomena examined are not influenced by measuring sensors. Furthermore, both visual perception of the phenomenon under examination and qualitative and quantitative analysis of the noticed results can be provided. One of the most sensitive, and one could say the most common methods, is the shadow and Schlieren method [2], [3], [4]. Both methods are based on changing the light refractive index in the environment under examination. This change is due to the existence of two or more different substances in the environment analyzed, or in the case of one substance under review by changing the density of the substance due to a change in pressure or temperature. The relationship between the light refraction index and density can be described for gaseous substances and with sufficient accuracy by applying the Lorenz-Lorentz Law [5].

$\frac{k^{2}-1}{k^{2}+2} \cdot \frac{1}{\rho}=$ const

where:

$$
\begin{aligned}
& \mathrm{k} \text { - the refractive index (1) } \\
& \rho \text { - the density }\left(\mathrm{kg} \cdot \mathrm{m}^{-3}\right)
\end{aligned}
$$

If we consider the value of the refractive index very close to one $(k \rightarrow 1)$, the relationship can be then simplified to

$$
\frac{(k-1)}{\rho}=\text { const }
$$

which is the law of Gladston-Dale [6].

The shadowgraph method scheme is shown in Figure 1. The ray passing through the nonhomogeneity in the layer under investigation is deflected in planes $x y$ and $x z$ with angles given by equations (3) and (4).

$$
\begin{aligned}
& \operatorname{tg} \varepsilon_{y}=\int \frac{1}{k} \cdot \frac{\partial k}{\partial y} d x \\
& \operatorname{tg} \varepsilon_{z}=\int \frac{1}{k} \cdot \frac{\partial k}{\partial z} d x
\end{aligned}
$$

where:

$$
\begin{aligned}
& \varepsilon_{y} \text { - the deflection angle in planes } x y\left({ }^{0}\right) \\
& \varepsilon_{z} \text { - the deflection angle in planes } x z\left({ }^{0}\right)
\end{aligned}
$$




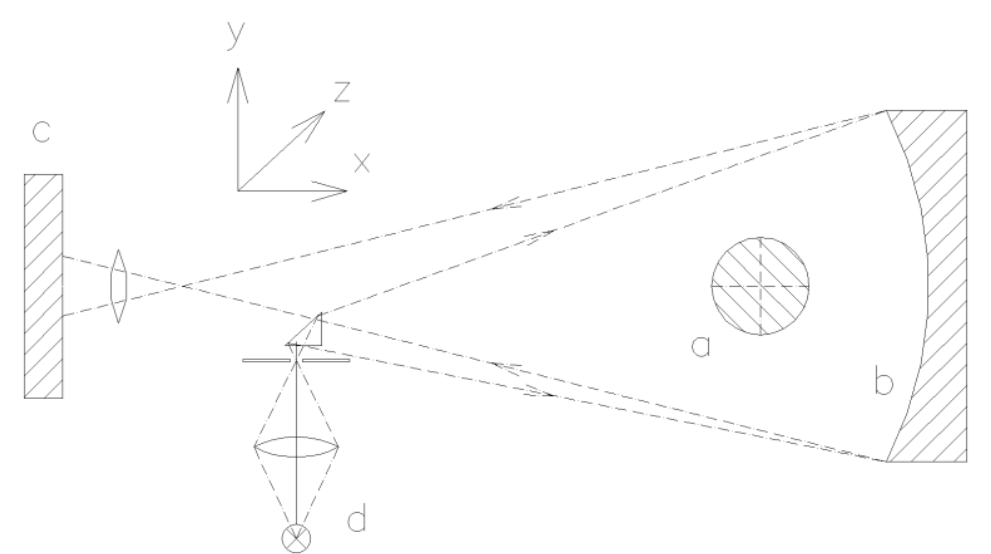

a) - free flow, b) - hollow mirror, c) - display plane, d) - spot light source

Figure 1 Shadowgraph method scheme

The rays passing through the area in which the density gradient is stable (the second density derivation equals to zero) is diverted by the same angle and, therefore, does not alter the lighting shield. In a place where the second density derivation is more than zero, the light rays diverge, and the lighting shield is reduced. On the contrary, in a space with a negative second density derivation, the rays converge and the lighting of the corresponding place on the shield increases.

Therefore, the change in the lighting shield is proportional to the second density derivation, where the constant value is determined by the geometry of the assembly and the value of the refractive index of the substance see equation (5).

$\frac{\Delta E}{E}=$ const $\cdot \frac{\partial^{2} \rho}{\partial y^{2}}+\frac{\partial^{2} \rho}{\partial z^{2}}$

where:

E the lighting, (lighting intensity) (Ix)

$\Delta \mathrm{E} \quad$ the change in lighting (lighting intensity) (Ix).

This method is suitable for displaying sharp changes in density, i.e. especially for impact waves and swirling areas.

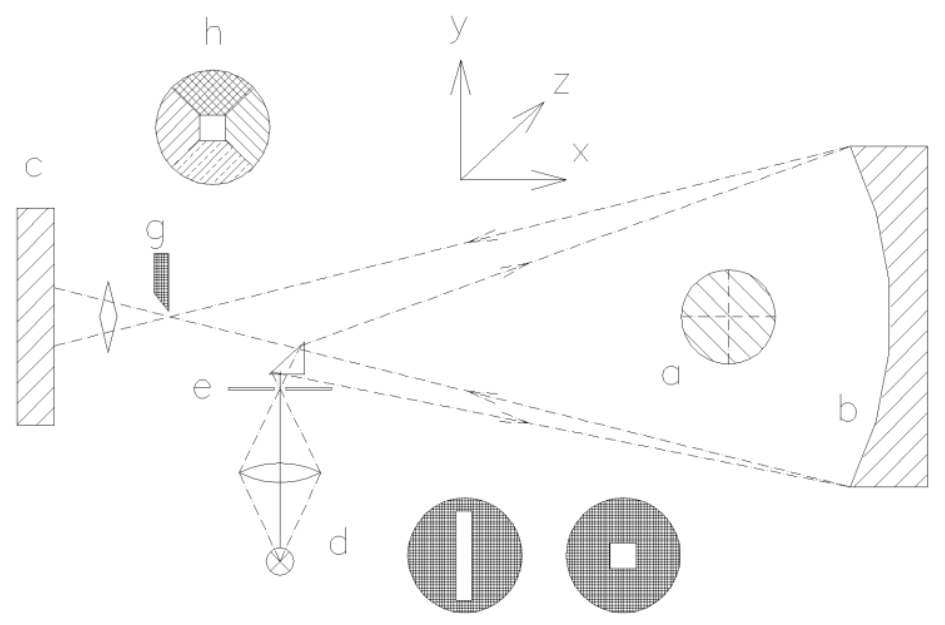

a) - free flow, b) - hollow mirror, c) - display plane, d) - spot light source, e) - screen for a classic black and white method, f) - screen for colour method, g) - optical knife, h) - colour segmental filter

Figure 2 Scheme of the Schlieren method 
The diagram of the Schlieren method is shown in Figure 2. The rays passing through the nonhomogeneity of the refractive index are again diffracted by angles given by the equations, as indicated in the shadowgraph method. The rays, previously passing outside the knife, are now stopped by a knife and some of the rays originally detained by the knife are now passing over its blade. The result is a display of nonhomogeneity on the shield, on which an image of the measuring space is also created. The change in the shield lightning here is proportional to the first density derivation and is affected by the geometry of the assembly and the insertion of the optical knife (Foucault knife) $\mathbf{g}$. The Schlieren method is suitable for displaying weak density changes.

Figure 3 is an example of an optical device solution [7] with which you can measure the shadowgraph method, classic and coloured Schlieren method, for which a colour segment filter is used instead of an optical knife. Thanks to it, the rays diffracted downwards in the picture are coloured in red, the rays diffracted upwards in blue, to the left - in green, and to the right - in yellow. Instead of a slit screen, for a colour Schlieren method, a screen with a hole is used, in which the image fits just into the colour segment filter viewport. From a colour photo, you can consider the directions and significations of changes in density gradients.

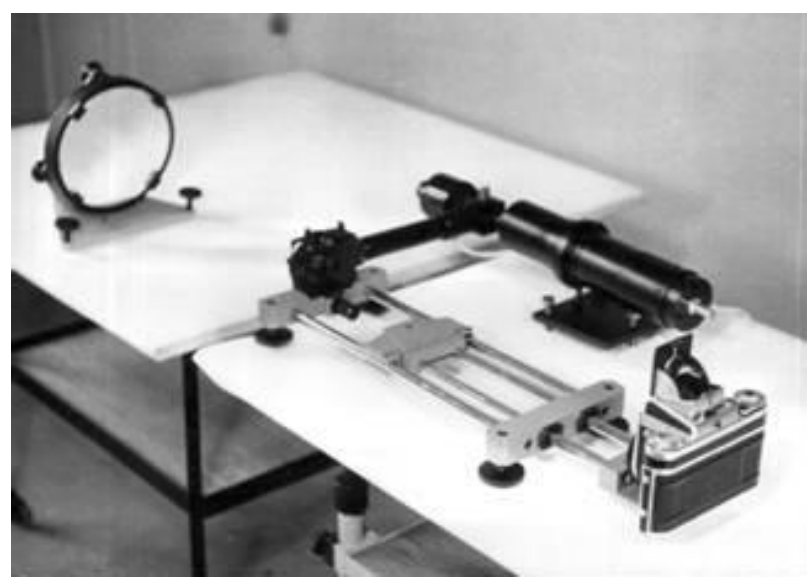

Figure 3 Alternative solution of optical equipment [7]

\section{SELECTED RESULTS OF THE OUTFLOW JETS VISUALISATION}

For the outflow jets visualisation were used the above-described methods. Below a representative selection of the outflow jets from lance (lance individual tuyeres) is displayed. Figure 4 shows pictures of the lance outflow jet structure at different total presses in front of the lance (nozzle).

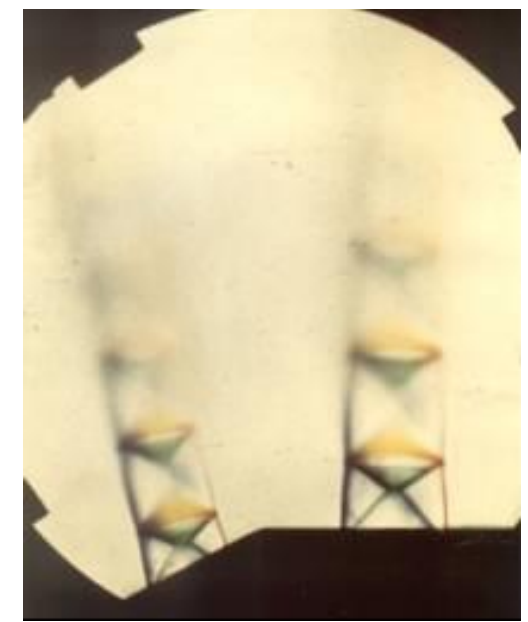

$\mathrm{p}_{0}=0.294 \mathrm{MPa}$

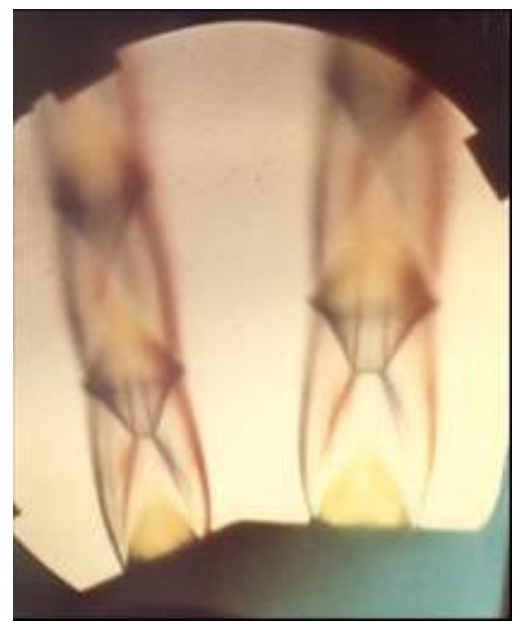

$\mathrm{p}_{0}=0.687 \mathrm{MPa}$

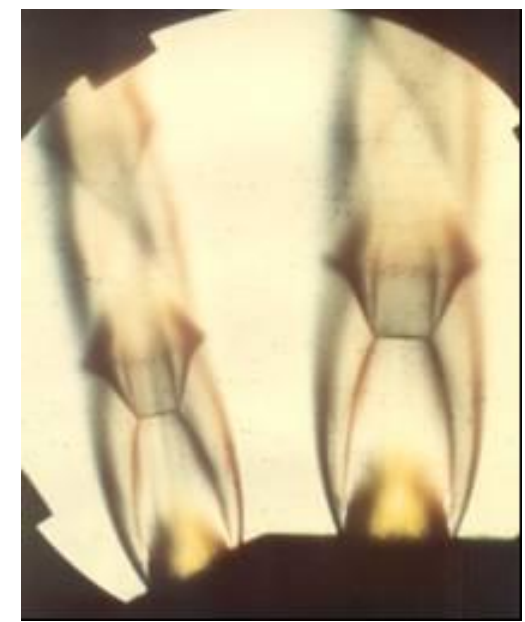

$\mathrm{p}_{0}=0.971 \mathrm{MPa}$

Figure 4 Photos are taken using the colour Schlieren method [7] 
The submitted photos were taken using a colour Schlieren method. When comparing the structure of individual outflow jets, there are visible changes through which the current passes when the total pressure $p_{0}$ is gradually increasing in front of the lance.

For comparison of black and white images for each method of outflow jet visualization, we also include the Figure $5 \mathrm{~A}, \mathbf{B}$, taken by the Schlieren and shadowgraph methods, for the highest pressure in front of the lance nozzles reaching $\mathrm{p}_{0}=0.971 \mathrm{MPa}$.

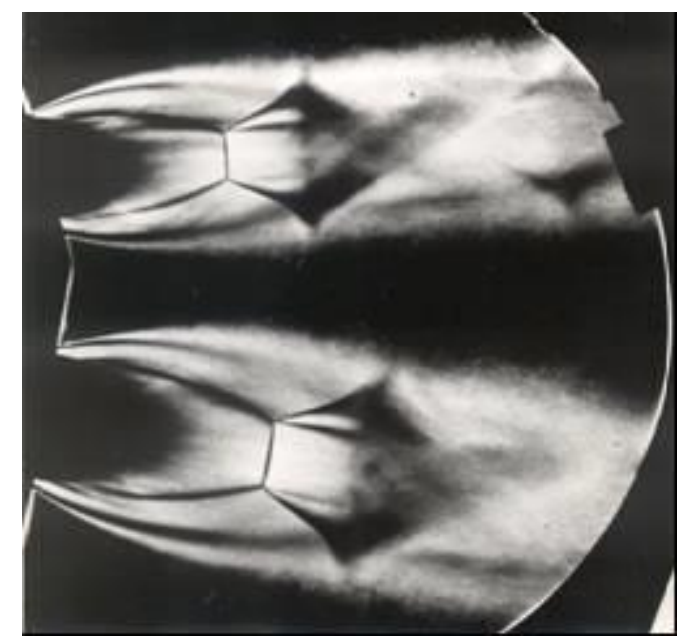

A - Black and white Schlieren method

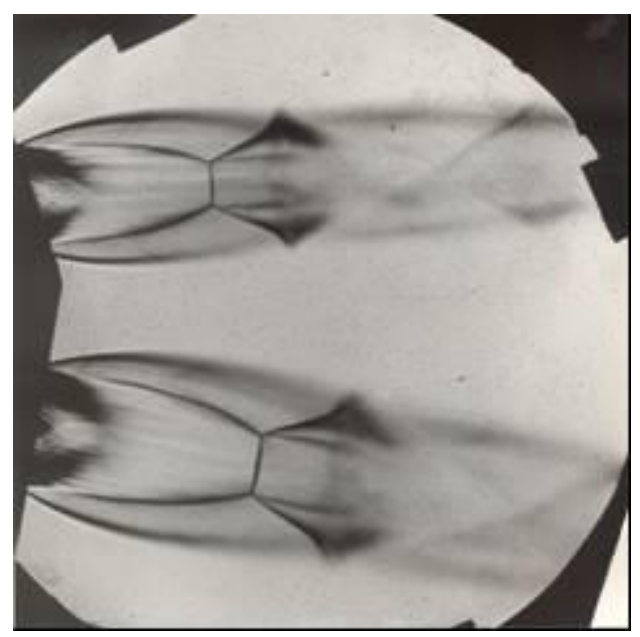

B - Shadowgraph method

Figure 5 Black and white images for $p_{0}=0.971 \mathrm{MPa}$ [7]

\section{CONCLUSION}

The design of individual components of the lance systems, especially the lance heads, significantly affects their proper functioning and the course of the technological process in high-temperature reactors. For flawless lance operation, it is important to observe the required parameters for the blown medium in front of the lance itself, as well as the design of the lance and individual nozzles. As regards the dimensions of lances and nozzles themselves, these can be designed based on calculation relationships that describe the correlation between the geometric dimensions of lances and nozzles and the individual state values. The possibility of verifying the real parameters of outflow jets from lances and nozzles gives designers valuable feedback based on which it is possible to correct future designs of individual components for the gas flow systems.

The possibility and means of visualization of the outflow jets allow this feedback to be realized to obtain valuable information about the real behaviour of a particular lance or nozzle design. Based on the analysis of the visualization results, it is possible to determine the points in which it is possible to measure the real values of the current state values.

\section{ACKNOWLEDGEMENTS}

This paper was created with the support of the SGS project at the Department of Production Machines and Design, Faculty of Mechanical Engineering VSB-TU Ostrava.

\section{REFERENCES}

[1] TOKER Gregory R. Holographic Interferometry: A Mach-Zehnder Approach. CRC Press, 2012, p. 211. ISBN: 9780367381646.

[2] RISTIĆ, S. Flow visualization techniques in wind tunnels -optical methods (Part II) [online]. [viewed 2019-11-24]. Available from: https://pdfs.semanticscholar.org/d790/8b03132c143837679d320383a6cf22cad7b9.pdf. 
[3] National Aeronautics and Space Administration. Schlieren system [online]. 2015 [viewed 2019-11-24]. Available from: https://www.grc.nasa.gov/www/k-12/airplane/tunvschlrn.html

[4] HU, H. Lecture 07: Flow Visualization techniques: Shadowgraph and Schlieren [online]. 2010 [viewed 2019-1124]. Available from: https://www.aere.iastate.edu/ huhui/teaching/2010Sx/AerE343L-AerE311L/Lecturenotes/Lecture-07-Notes.pdf.

[5] KADLEC, K. Měření optických vlastností kapalin (část 1.) Listy cukrovarnické a řepařské. 2018, vol. 134, no. 9 10, pp. 348-351.

[6] MERZKIRCH, W. Visualization of flow [online]. 2011 [viewed 2019-11-24]. Available from: http://www.thermopedia.com/content/1245/

[7] MIKOLAJEK, J. CHAMRÁD, S. et al. Studium aerodynamiky zkůjňovacích pochodů. Ostrava, 1977. p. 63. Výzkumná zpráva. Faculty of Mechanical Engineering, VSB - Technical University of Ostrava. 